\title{
Questionário de Crenças Irracionais e Escala de Crenças Irracionais: Propriedades Psicométricas
}

\author{
Elisa Medici Piz̨ão Yoshida ${ }^{12}$ \\ Pontifícia Universidade Católica de Campinas \\ Fernando Antonio Basile Colugnati \\ Universidade Federal de São Paulo
}

\begin{abstract}
Resumo
São estimadas e comparadas as propriedades psicométricas de versões em português de duas medidas de auto-relato para crenças irracionais: Questionário de Crenças Irracionais (QCI) e Escala de Crenças Irracionais (ECI). Oitocentos e quarenta e nove universitários completaram os instrumentos, além do Questionário de Saúde Geral de Goldberg (QSG) usado como critério externo da validade discriminante. Os resultados mostram que os dois instrumentos têm boa consistência interna e fidedignidade de teste e reteste, mas nenhum deles correlaciona-se significantemente com o QSG. Análise do componente principal da ECI indica estrutura de fator único com 16 dos 20 itens da escala apresentando altas cargas num mesmo fator para ao menos um dos sexos e a amostra geral. Os demais itens necessitarão ser adaptados ou modificados. Recomenda-se que a ECI seja preferida ao QCI na avaliação de crenças irracionais de sujeitos brasileiros e que novas pesquisas de validade sejam realizadas. Palavras-chave: Crenças irracionais; avaliação de crenças irracionais; propriedades psicométricas; validade; precisão.
\end{abstract}

Irrational Beliefs Questionnaire and Irrational Belief Scale: Psychometric Properties

\begin{abstract}
The study aimed to estimate and compare the psychometric properties of the Portuguese versions of two irrational-beliefs selfreport measures: the Irrational Belief Questionnaire (IBQ) and the Irrational Belief Scale (IBS). Eight hundred fourty nine college students completed both instruments besides the Goldberg's General Health Questionnaire (GHQ), used as external criterion of the discriminant validity. Results show that both instruments have good internal consistency and test-retest reliability, but none of them correlated significantly with the GHQ. The IBS principal component analysis points to a singlefactor structure with 16 out of the 20 scale's items high loading on the same factor in at least one of the groups according to the sex and the general sample. It is suggested that the IBS should be preferred to the IBQ for assessing irrational beliefs of Brazilians subjects and that further research on validity should be carried out .

Keywords: Irrational beliefs; irrational beliefs assessment; psychometric properties; validation; reliability.
\end{abstract}

O estudo teve como objetivo estimar e comparar as propriedades psicométricas de versões em português de dois instrumentos desenvolvidos para avaliar crenças irracionais: o Irrational Belief Questionnaire - IBQ (Newmark, Frerking, Cook \& Newmark, 1973), aqui denominado de Questionário de Crenças Irracionais on QCI, e a Irrational Belief Scale - IBS (Malouff \& Schutte,1986), aqui chamada de Escala de Crenças Irracionais, ou ECI.

As crenças irracionais são o foco da Terapia Racional Emotiva - RET, recentemente renomeada, Terapia do Comportamento Racional Emotivo - REBT (Ellis, 1995). Está fundada na chamada Teoria $\mathrm{ABC}$ dos distúrbios humanos, segundo a qual o $A$ corresponde a eventos ativadores indesejáveis de experiências (Activating events of

\footnotetext{
${ }^{1}$ Endereço para correspondência: Av. Francisco Dinis,227, 06030-380, Osasco, SP. Fone/Fax: (11) 36841497. E-mail: eyoshida.tln@terra.com.br ${ }^{2}$ Os autores agradecem a colaboração da Dra. Marilda Emmanuel Novaes Lipp na tradução dos instrumentos e à bolsista de IC/FAPESP, Alessandra Gonçalves Martão, pela colaboração na coleta e tabulação dos dados.
}

experiences), o $B$ a crenças (Beliefs) e o $C$ a conseqüências cognitivas, emocionais e comportamentais. Frente a $A$ a pessoa pode reagir com crenças racionais ou irracionais, criando conseqüências emocionais e comportamentais apropriadas - aC com suas crenças racionais ou conseqüências inapropriadas e disfuncionais, com suas crenças irracionais - iC (Ellis, 1993).

Para definir o que entendia por crenças irracionais, Ellis (1993) listou as crenças mais encontradas na prática clínica, reduzindo-as posteriormente a poucas categorias com vários derivados. Esta definição, dita ostensiva do constructo, suscitou algumas críticas, já que diferentes listas propostas continham sobreposições, algumas crenças listadas teriam sido consideradas inadequadas pelo próprio Ellis, além de poderem variar no tempo para um mesmo juiz, ou ser difícil concordar-se quanto a quem deva ser este juiz (Rorer, 1989a, 1989b).

A dificuldade de se definir crenças irracionais encontrase diretamente relacionada à maneira de estudá-las 
empiricamente, sendo que algumas tentativas de se criar instrumentos para medi-las não revelaram validade discriminante adequada, como por exemplo, o Rational Behavior Inventory - RBI (Shorkey \& Whiteman, 1977) ou o Questionário de Crenças Irracionais - QCI, proposto por Newmark e colaboradores (1973).

O RBI (Shorkey \& Whiteman, 1977) foi desenvolvido com o propósito de avaliar os pacientes e planejar e avaliar RETs. É um inventário de tipo self-report, com respostas em escala Likert de 5 pontos, constituído de 38 itens distribuídos por 11 fatores (sub-escalas), sendo que um mesmo item aparece em duas sub-escalas. Elas se referem respectivamente a: 1) avaliação da seriedade de situações frustrantes; 2) sentimentos de culpa e atribuição de culpa a outros por desvios de costumes e valores tradicionais; 3) demanda por perfeccionismo em todas as áreas do comportamento; 4) quadro de referências do qual o indivíduo faz juízo de valor sobre seus atributos, idéias e comportamentos; 5) demanda de que todas as pessoas preocupem-se e ajudem umas às outras; 6) tendência a responsabilizar e culpar a si e a outros por enganos, pecados e erros; 7) habilidade para conter tendência de esquiva aceitando dificuldades e trabalho em tarefas desprazíveis; 8) aceitação de independência em tomada de decisão e aceitação de conseqüências de ações ou decisões; 9) tendência a se perturbar com avaliações negativas de atributos pessoais e circunstâncias de vida; 10) tendência a se perturbar com possíveis infortúnios futuros, independentemente da probabilidade de sua ocorrência; 11) crenças relacionadas ao controle sobre as emoções.

Para explorar a validade do RBI, os autores aplicaramno, inicialmente, em 40 profissionais que realizavam workshop de um dia sobre RET, conduzido por Albert Ellis. Esperavam que os participantes apresentassem escores mais altos ao final do workshop, quando comparados aos obtidos antes do seu início. No entanto, apesar de se tratarem de profissionais e de terem respondido ao inventário imediatamente após as conferências sobre RET, obtiveram diferença significante na direção esperada ao nível de 0,025 (teste $t$ uni-caudal) apenas para os escore total e para a sub-escala 5 (demanda por cuidados e ajuda). Num segundo estudo, envolvendo 87 profissionais que realizavam workshop de dois dias sobre RET, também conduzido por Ellis, houve diferenças significantes na direção predita ao nível de 0,005 ou acima (teste $t$ unicaudal), para os escores total e para as sub-escalas 1, 2, 3, 4, 5, 6, 8, 9 e 11. E ao nível de 0,025 para a sub-escala 10 .

As diferenças observadas entre as duas amostras levaram os autores a suporem que medidas de racionalidade tendem a ser maiores com maior exposição a experiências relevantes de aprendizado, mas que novas pesquisas de validade deveriam ser conduzidas.

Quanto ao QCI (Newmark \& cols., 1973), composto por 11 frases afirmativas referentes às crenças irracionais, é uma versão algo modificada do Questionário de Crenças Irracionais de Ellis, de MacDonald e Games (1972, citado em Newmark \& cols., 1973). Para cada afirmação o sujeito deve escolher entre a alternativa verdadeira ou falsa, sendo que quanto mais respostas positivas às afirmações mais irracional será o funcionamento emocional do indivíduo e, por conseguinte, mais comprometida sua adaptação.

Para verificar a validade discriminante, o QCI foi aplicado a três grupos de sujeitos dos quais: dois formados por pacientes internados em hospital psiquiátrico, com sintomatologia neurótica $(N=120)$ e com padrões de ajustamento caracterológico $(n=98)$, segundo o MMPI, e, um grupo $(N=120)$ considerado normal, constituído por estudantes universitários. Os qui-quadrados entre os grupos indicaram que o grupo neurótico acatou quatro das crenças irracionais, em contraste com os grupos normal e com desordem de personalidade, que não acataram nenhuma delas. Além disto, o grupo neurótico demonstrou uma freqüência significante maior de aceitação de 8 das 11 crenças, quando comparado tanto ao grupo de normais quanto ao caracterológico. E estes dois últimos, apresentaram freqüência de rejeição bastante semelhantes em relação aos 11 itens.

Apesar das limitações evidenciadas por este instrumento, ele já foi utilizado em nosso meio em algumas pesquisas como, por exemplo, a de Calais (1997) que investigou a relação entre crenças irracionais e habilidade social, inferida pelo número de escolhas/rejeição em avaliações sociométricas envolvendo um grupo de estudantes universitários, ou a de Pinto (1996), em que a existência de crenças irracionais foi considerada uma das fontes de stress em pacientes com vitiligo.

Um terceiro instrumento, empiricamente construído, é a Escala de Crenças Irracionais-ECI, desenvolvida por Malouff e Schutte (1986). Segundo os autores, houve a preocupação de superar as dificuldades com a validade discriminante verificada com os instrumentos anteriores, descartando-se itens em que reações de ansiedade e de depressão pudessem estar envolvidas (Schutte \& Malouff, 1995).

Para o estabelecimento da validade da ECI, Malouff e Schutte (1986) avaliaram sua consistência interna $(a l p h a=0,80)$ com uma amostra de 300 universitários, dos quais 192 eram homens e 108 mulheres (idade média= 18,76 e $d p=1,87$ ), não tendo sido observada diferença significante nos escores médios de homens e mulheres. Para a precisão de teste e reteste, reaplicaram o instrumento a uma amostra de 44 sujeitos retirados da anterior (23 homens e 21 mulheres), após um intervalo de duas semanas, tendo obtido 
coeficientes de correlação produto-momento para os dois sexos igual a 0,89 .

Em relação à validade, estudos indicaram associação da escala com outras medidas de crenças irracionais. Por exemplo, estimou-se os coeficientes de correlação produtomomento entre a ECI e o Irrational Beliefs Test de Jones (1969, citado em Malouff \& Schutte, 1986), em amostra composta por 31 homens e 25 mulheres, estudantes de psicologia, com idade média de 20,20 anos e $d p=4,30$, tendose obtido $r(55)=0,55, p<0,001$. Além disto, os autores solicitaram aos 300 sujeitos referidos no estudo da consistência interna, para que completassem a Social Desirability Scale de Crowne e Marlowe (1964, citado em Malouff \& Schutte, 1986), um instrumento de 33 itens, teoricamente não relacionado a crenças irracionais. Do total, 243 sujeitos responderam a todos os itens das duas escalas cujo coeficiente de correlação produto-momento resultante foi igual a $r(242)=-0,27, p<0,001$. Ou seja, escores de alta sociabilidade correlacionaram-se com baixos escores de crenças irracionais.

Em outro estudo estimou-se a associação das crenças irracionais com depressão (Malouff \& Schutte, 1986). A amostra foi constituída de 47 pacientes (19 homens) que procuravam tratamento para depressão e 6 não-pacientes ( 3 homens), estudantes universitários. A idade média dos pacientes era de 38,89 anos, $d p=8,57$ enquanto que dos não-pacientes de 35,67 anos e $d p=3,33$. A medida de depressão usada, realizada de forma independente por um amigo íntimo ou parente do sujeito, consistiu numa escala de avaliação comportamental da depressão (Malouff, 1984, citado em Malouff \& Schutte, 1986), contendo nove índices comportamentais de depressão, como por exemplo "expressão facial triste". Obteve-se associação significante entre os escores de crenças irracionais e os de depressão para toda a amostra $(r(52)=0,30, p<0,02)$, assim como para o grupo de pacientes $(r(46)=0,22, p<0,08)$.

Outros estudos estimaram a associação entre depressão e ansiedade (Templeman, 1990), depressão e bulimia (Wertheim \& Poulakis, 1992), ansiedade e hostilidade (Malouff, Valdenegro \& Schutte, 1987), neuroticismo (Warren \& Zgourides, 1989), ou ainda, distúrbios de alimentação (Mayhew \& Edelman, 1989), sendo que todos eles indicaram associações significantes com a ECI.

$\mathrm{O}$ acúmulo de evidências fornecidas pelas pesquisas citadas sugere que dentre os instrumentos disponíveis para uma eventual adaptação para o português, a ECI seria a que apresenta melhor validade enquanto instrumento de avaliação de crenças irracionais. Por outro lado, apesar de haver indicações na literatura de que o QCI careceria de validade discriminante (Newmark \& cols., 1973), considerou- se que a verificação de suas qualidades psicométricas junto a amostras brasileiras não deveria ser descartada, já que medidas empíricas são sujeitas a diferenças em populações diversas ou mesmo em uma mesma população, quando se compara medidas realizadas em períodos de tempo diferentes (Anastasi \& Urbina, 2000).

Considerando portanto o fato de que o QCI já vem sendo utilizado em nosso meio, decidiu-se aferir sua consistência interna, precisão de teste e reteste, além de medidas de validade, buscando uma adaptação deste instrumento à nossa realidade. Ademais, julgou-se que um estudo comparativo das qualidades psicométricas deste instrumento com as de outro, que já tivesse se revelado anteriormente superior em pesquisas estrangeiras, poder-se-ia constituir em contribuição original. Neste sentido, selecionou-se o QCI e a ECI como instrumentos de investigação psicométrica para o presente estudo.

Como medida de critério externa para a medida da validade discriminante optou-se pelo Questionário de Saúde Geral de Goldberg - QSG (Pasquali, Gouveia, Andriola, Miranda \& Ramos, 1996), uma vez que o comprometimento do funcionamento emocional tem se mostrado na prática clínica associado ao uso de crenças irracionais. Por outro lado, é um instrumento que dispõe de adaptação brasileira realizada com a utilização de amostra de universitários, semelhante a do presente estudo.

\section{Método}

\section{Participantes}

A amostra é constituída de 849 universitários dos cursos de psicologia, fonoaudiologia, contabilidade superior e administração de empresas, de universidade particular do estado de São Paulo, sendo os dois primeiros cursos diurnos e os dois últimos, noturnos. Quanto ao sexo, $64,4 \%$ são femininos $(n=547)$ e $35,6 \%$ masculinos $(n=302)$. Dos 838 que informaram sobre a situação empregatícia, $61,2 \%$ trabalham $(n=514)$. Em relação ao estado civil, 807 informaram sua condição e encontram-se assim distribuídos: $88,8 \%$ são solteiros $(n=717), 10 \%$ casados/amigados $(n=81), 1 \%$ separado/divorciado $(n=8)$ e $0,18 \%$ viúvo $(n=1)$. Quanto à faixa etária predominam os jovens, com média de 21,3 anos $(d p=3,98)$, com mínimo de 18 e máximo de 51.

Vale observar que embora não se tenha obtido dados quanto à classe econômica dos participantes, o fato de se tratarem de estudantes de universidade particular sugere que sejam, em sua maioria, jovens de classe média e média/ baixa, já que $61,2 \%$ trabalham e a maioria é solteira. Por estas características é plausível pensar que a amostra 
represente adequadamente os estudantes universitários brasileiros.

\section{Instrumentos}

Questionário de Crenças Irracionais - QCI (Newmark \& cols., 1973) - consiste de 11 frases afirmativas que se referem a crenças irracionais propostas por Albert Ellis, às quais $\mathrm{O}$ sujeito deve responder com as alternativas $\mathrm{V}$ (verdadeiro) ou F (falso). Quanto maior o número de Vs assinalados mais irracional é considerado o seu funcionamento emocional.

Escala de Crenças Irracionais - ECI (Malouff \& Schutte, 1986; Schutte \& Malouff, 1995 ) - composta de 20 itens aos quais o sujeito deve responder através de uma escala Likert de 5 pontos que vai de discordo fortemente (1), a concordo fortemente (5). A soma de pontos pode variar entre 20 e 100 pontos, estando os maiores índices associados a mais crenças irracionais.

Questionário de Saúde Geral de Goldberg - QSG (adaptação brasileira)(Pasquali \& cols., 1996) - foi desenvolvido com o propósito de identificar o perfil sintomático de saúde metal de pessoas com distúrbios psiquiátricos não extremados. De tipo self-report, consta de 60 itens aos quais o sujeito deve responder através de escala de tipo Likert de 4 pontos, em que as alternativas podem variar um pouco em função do teor da pergunta. A avaliação realizada com a ajuda de um crivo ou de computador se dá inicialmente através da obtenção do escore bruto de cada um dos cinco fatores (1. stress psíquico, 2. desejo de morte, 3. desconfiança no desempenho, 4. distúrbios do sono e 5. distúrbios psicossomáticos), bem como do escore bruto geral do questionário. A seguir, obtém-se os escores sintomáticos dividindo-se a soma das respostas que compõem o fator pelo número de itens que o fator tem. Protocolos com $10 \%$ ou mais questões não respondidas são desprezados. Uma tabela de escores percentílicos indica o perfil dos sintomas de distúrbios de saúde do sujeito em relação à norma.

\section{Procedimentos}

Uma doutora em psicologia, fluente em inglês, e a primeira autora procederam à tradução para o português dos dois instrumentos, de forma independente. A seguir, comparou-se as traduções e se discutiu algumas expressões que poderiam tornar os instrumentos mais compreensíveis para sujeitos brasileiros, até a obtenção das formas utilizadas (os interessados poderão obter o instrumento com a primeira autora).

A aplicação para a coleta dos dados deu-se na sala de aula dos participantes, tendo-se aplicado primeiro o QSG e a seguir o ECI ou o QCI, de forma alternada. Em horário previamente combinado com o professor, a bolsista de Iniciação Científica apresentava-se à classe, informava sobre os objetivos da pesquisa, esclarecia tratar-se de colaboração voluntária e enfatizava a preservação do anonimato dos sujeitos. Além disso, informava sobre a possibilidade de uma segunda aplicação dos instrumentos com fins de reteste, após um mês. Para aqueles que concordavam em participar, pedia o preenchimento de um formulário de Consentimento Livre e Esclarecido. Aos que se recusavam, solicitava que se retirassem da sala antes do início da aplicação dos instrumentos.

O reteste foi realizado depois de um mês, para apenas parte da amostra $(n=87)$.

\section{Resultados}

\section{Consistência Interna}

Para estimar a consistência interna dos instrumentos foram utilizados o alpha de Cronbach e a correlação média inter-item, calculados para ambos os sexos e a amostra geral. Para a ECI utilizou-se a correlação por postos de Spearman e para o QCI, por se tratarem de respostas dicotômicas (V ou F, ou 1 e 0 respectivamente), utilizou-se a correlação Tetracórica, adequada para este tipo de dado.

Para a ECI, o alpha masculino é ligeiramente mais alto que o feminino, respectivamente, 0,78 e 0,76 , enquanto que para a amostra geral é 0,73 . A correlação inter-item é fraca para as duas subamostras (0,15 para sujeitos masculinos e 0,13 para femininos), assim como para a amostra geral $(0,14)$, e tal fato será evidenciado na análise fatorial.

Para o QCI a diferença entre os sexos é um pouco maior, $0,74(\mathrm{M})$ e $0,70(\mathrm{~F})$, e o índice geral, menor $(0,71)$. As correlações médias se apresentaram um pouco mais altas que na ECI (0,21/M; 0,19/F.; 0,20/geral), mas ainda muito fracas.

\section{Precisão de Teste-Reteste}

A precisão de teste reteste foi feita com 87 participantes dos quais apenas 11 do sexo masculino. Para a ECI os índices de reteste foram: 0,88 para a amostra masculina e 0,82 tanto para a feminina quanto geral. Quanto ao QCI, o índice de reteste para a amostra feminina foi 0,78 e geral 0,72 . Para o sexo masculino não houve variabilidade das respostas $(100 \%$ replicadas), indicando a estabilidade da medida no tempo.

\section{Validade de Constructo: Estudo 1}

A Tabela 1 apresenta as medidas resumo para o escore geral do QSG (qsgG), os cinco escores das dimensões de sintomas do QSG (qsg1, ... , qsg5), a soma dos pontos da ECI (esceci) e a soma de V do QCI (escqci). Nota-se 
Tabela 1

Resumo dos Escores do QSG, ECI e QCI, por Sexo e no Geral

\begin{tabular}{|c|c|c|c|c|c|}
\hline Variável & $N$ & $m$ & $d p$ & Mínimo & Máximo \\
\hline \multicolumn{6}{|c|}{ Sexo Masculino } \\
\hline Qsg G & 302 & 1,74 & 0,36 & 1 & 3,37 \\
\hline Qsg 1 & 302 & 1,94 & 0,56 & 0,11 & 3,77 \\
\hline Qsg 2 & 302 & 1,26 & 0,48 & 0,11 & 4 \\
\hline Qsg 3 & 302 & 1,92 & 0,44 & 1 & 3,47 \\
\hline Qsg 4 & 302 & 1,45 & 0,49 & 0,13 & 3,5 \\
\hline Qsg 5 & 302 & 1,62 & 0,40 & 1 & 3,5 \\
\hline Esceci & 300 & 59,47 & 11,39 & 21 & 95 \\
\hline Escqci & 291 & 3,87 & 2,11 & 0 & 11 \\
\hline \multicolumn{6}{|c|}{ Sexo Feminino } \\
\hline Qsg G & 547 & 1,83 & 0,38 & 0,18 & 3,75 \\
\hline Qsg 1 & 547 & 2,08 & 0,59 & 0,15 & 4 \\
\hline Qsg 2 & 547 & 1,32 & 0,49 & 0,11 & 3,75 \\
\hline Qsg 3 & 547 & 1,99 & 0,46 & 0,16 & 4,12 \\
\hline Qsg 4 & 547 & 1,59 & 0,60 & 0,16 & 4 \\
\hline Qsg 5 & 547 & 1,78 & 0,43 & 1 & 3,7 \\
\hline Esceci & 543 & 59,78 & 10,28 & 26 & 87 \\
\hline Escqci & 535 & 3,50 & 1,94 & 0 & 10 \\
\hline \multicolumn{6}{|l|}{ Geral } \\
\hline Qsg G & 849 & 1,80 & 0,38 & 0,18 & 3,75 \\
\hline Qsg 1 & 849 & 2,03 & 0,58 & 0,11 & 4 \\
\hline Qsg 2 & 849 & 1,30 & 0,48 & 0,11 & 4 \\
\hline Qsg 3 & 849 & 1,97 & 0,46 & 0,16 & 4,12 \\
\hline Qsg 4 & 849 & 1,54 & 0,57 & 0,13 & 4 \\
\hline Qsg 5 & 849 & 1,72 & 0,42 & 1 & 3,7 \\
\hline Esceci & 843 & 59,67 & 10,68 & 21 & 95 \\
\hline Escqci & 826 & 3,63 & 2,01 & 0 & 11 \\
\hline
\end{tabular}

que não há uma diferença muito grande entre as médias e os $d p s$ nos sexos masculino e feminino, mostrando uma certa estabilidade destes escores quanto ao sexo.

A Tabela 2 apresenta os coeficientes de correlação de Spearman dos escores estudados. Nota-se que as correlações só são consideráveis entre os escores do QSG, que variam entre 0,56 e 0,86, constituindo-se em um indicador da validade convergente deste instrumento (Anastasi \& Urbina, 2000). As que envolvem os escores da ECI e do QCI e o QSG, contrariamente ao esperado, mostram correlação presente, porém fraca.

Tabela 2

Matriz de Correlação de Spearman entre os Escores

\begin{tabular}{llllllll}
\hline QSG_ & QSG_0G & QSG1 & QSG2 & QSG3 & QSG 4 & QSG 5 & ESCECI \\
\hline QSG_1 & 0,86 & & & & & & \\
QSG_2 & 0,69 & 0,59 & & & & & \\
QSG_3 & 0,83 & 0,68 & 0,55 & & & & \\
QSG_4 & 0,56 & 0,46 & 0,36 & 0,32 & & & \\
QSG_5 & 0,73 & 0,67 & 0,41 & 0,54 & 0,43 & & \\
ESCECI & 0,35 & 0,37 & 0,30 & 0,25 & 0,18 & 0,24 & \\
ESCQCI & 0,16 & 0,16 & 0,15 & 0,11 & 0,08 & 0,08 & 0,47 \\
\hline
\end{tabular}


No que diz respeit0 ao grau de associação entre a ECI e o QCI é apenas moderada $(0,47)$ indicando que estes instrumentos estariam medindo constructos algo diferentes entre si.

\section{Validade de Constructo: Estudo 2}

Este tópico trata apenas da análise fatorial (AF) da ECI, já que devido à natureza dicotômica dos dados do QCI, os métodos clássicos de $\mathrm{AF}$ não se empregam. A dificuldade computacional das técnicas alternativas, como por exemplo a AF sobre a matriz de correlação estimada pelos coeficientes de correlação tetracórica, inviabilizaram este tipo de análise.

As análises com a ECI foram realizadas em cada sexo e de forma geral a fim de se observar a invariância da estrutura.

A Figura 1 apresenta os autovalores das amostras: masculina, feminina e geral. A presença de um fator único é
Pode-se dizer que existe uma estrutura de fator único, logo a escala é basicamente unidimensional e que esta varia de forma não muito marcante, apesar de se mostrar mais sensível no sexo masculino.

A Tabela 4 apresenta as cargas fatoriais para cada item. Observa-se novamente que a estrutura no sexo masculino é mais marcante no primeiro fator que as demais (feminino e geral).

Apesar do desequilíbrio das amostras quanto ao sexo, os coeficientes de consistência interna da ECI são muito semelhantes: 0,78 (M) e 0,76 (F). Em ambos os casos, encontram-se acima de 0,75 , considerado adequado para este tipo de instrumento. Quanto ao a geral $(0,73)$, sugere boa confiabilidade para a escala, apesar de inferior ao obtido por Malouff e Schutte (1986) $(a=0,80)$, em pesquisa que

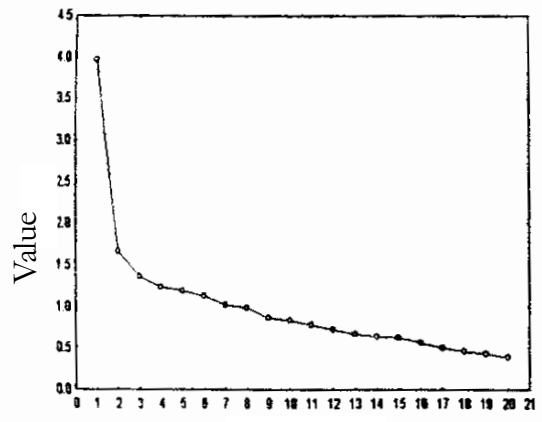

Number of Eigenvalues

(a)

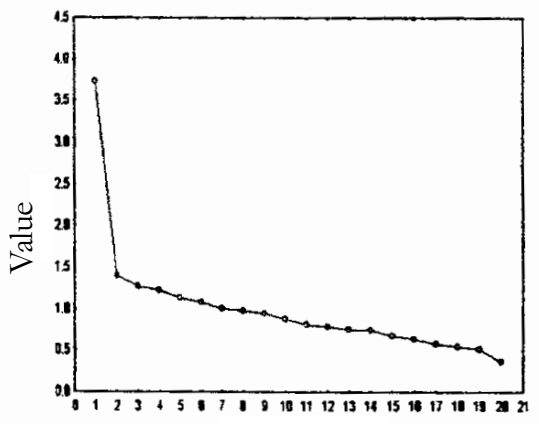

(b)

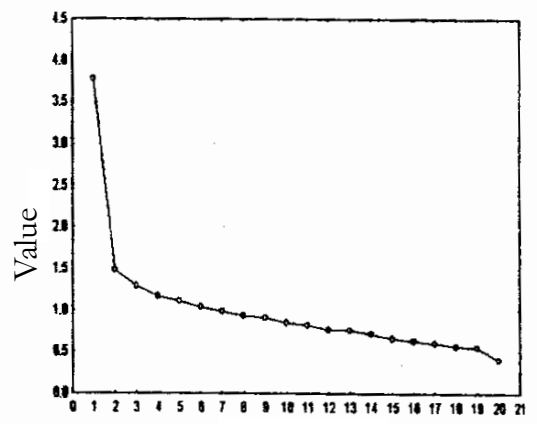

Number of Eigenvalues

(c)

Figura 1. Representação gráfica dos autovalores da análise fatorial, (a) masculino, (b) feminino, (c) geral.

Tabela 3

Autovalores da Análise Fatorial por Sexo e no Geral, da ECI

\begin{tabular}{lcc}
\hline ECI & Autovalores $\left(1^{\circ}\right.$ e $\left.2^{\circ}\right)$ & $\%$ acumulada da Var. \\
\hline Masculino & 3,97 & 19,85 \\
\multirow{2}{*}{ Feminino } & 1,67 & 28,18 \\
& 3,73 & 18,65 \\
Geral & 1,40 & 25,63 \\
& 3,77 & 18,87 \\
& 1,48 & 26,27 \\
\hline
\end{tabular}

evidente nos três casos, apesar de não muito forte, sendo que para a do sexo masculino a queda das cargas fatoriais é mais marcante. A baixa correlação inter-item, apresentada anteriormente, pode explicar este fato.

A Tabela 3 mostra os dois primeiros autovalores e a percentagem de explicação destes acumulada. O segundo fator é apresentado apenas como auxílio e identificação de itens que por algum motivo se apresentam com boa carga. contou com amostra de estudantes de psicologia norteamericanos $(N=300)$. A este respeito, é possível cogitar que nuances de natureza semântica podem conferir, a alguns itens, sentidos algo diversos da versão original, o que estaria se refletindo no grau de consistência interna do instrumento. Estas interferências podem igualmente estar presentes nas cargas fatoriais dos itens, como se verá mais à frente. 
Tabela 4

Cargas Fatoriais (2 primeiros fatores), por Sexo e no Geral, para a ECI

\begin{tabular}{lllllll}
\hline Grupo & \multicolumn{2}{c}{ Masculino } & \multicolumn{2}{c}{ Feminino } & \multicolumn{2}{c}{ Geral } \\
\hline Item & Fator 1 & Fator 2 & Fator 1 & Fator 2 & Fator 1 & Fator 2 \\
\hline 1 & 0,346 & 0,118 & 0,277 & 0,275 & 0,295 & $-0,253$ \\
2 & 0,481 & $-0,114$ & 0,287 & 0,156 & 0,365 & $-0,151$ \\
3 & 0,407 & 0,049 & 0,377 & 0,156 & 0,387 & $-0,102$ \\
4 & 0,401 & $-0,191$ & 0,386 & 0,420 & 0,374 & $-0,447$ \\
5 & 0,400 & $-0,382$ & 0,408 & 0,296 & 0,400 & $-0,292$ \\
6 & 0,467 & $-0,154$ & 0,507 & $-0,276$ & 0,491 & 0,126 \\
7 & 0,486 & $-0,108$ & 0,530 & $-0,218$ & 0,518 & 0,120 \\
8 & 0,639 & 0,053 & 0,625 & $-0,162$ & 0,626 & 0,126 \\
9 & 0,164 & $-0,540$ & 0,182 & 0,590 & 0,170 & $-0,612$ \\
10 & 0,469 & 0,172 & 0,323 & $-0,319$ & 0,385 & 0,280 \\
11 & 0,397 & $-0,419$ & 0,449 & 0,073 & 0,433 & $-0,190$ \\
12 & 0,379 & 0,172 & 0,511 & 0,066 & 0,450 & $-0,077$ \\
13 & 0,403 & $-0,146$ & 0,483 & $-0,279$ & 0,452 & 0,132 \\
14 & 0,577 & 0,057 & 0,654 & $-0,087$ & 0,624 & 0,125 \\
15 & 0,449 & 0,469 & 0,346 & $-0,037$ & 0,389 & 0,250 \\
16 & 0,622 & 0,196 & 0,459 & $-0,006$ & 0,526 & 0,140 \\
17 & 0,372 & $-0,237$ & 0,364 & 0,038 & 0,373 & $-0,034$ \\
18 & 0,415 & 0,386 & 0,439 & $-0,051$ & 0,432 & 0,217 \\
19 & 0,201 & $-0,562$ & 0,195 & 0,538 & 0,192 & $-0,599$ \\
20 & 0,536 & 0,294 & 0,478 & $-0,136$ & 0,502 & 0,178 \\
& & & & & & \\
\hline
\end{tabular}

Quanto ao QCI, verifica-se que os a da amostra geral $(0,71)$ e das amostras por sexo $(0,74$ e 0,70$)$ encontramse abaixo dos obtidos para a ECI, o que fala a favor daquela escala como instrumento mais preciso em nosso meio. Além disto, não foram encontrados outros estudos voltados para a estimativa da fidedignidade do QCI, que permitissem a comparação da consistência com a obtida em outras situações sócio-culturais.

Quanto à precisão de teste e reteste, segundo o sexo, ficou bastante prejudicada pelo desequilíbrio numérico das amostras. Porém, os índices com a amostra geral sugerem boa constância no tempo para os dois instrumentos ainda que, também neste caso, a ECI $(0,82)$ tenha se mostrado superior ao QCI $(0,72)$.

Comparando-se o valor obtido do coeficiente momento-produto da ECI $(r=0,82)$ com o de Malouff e Schutte $(1986)(r=0,89)$, poder-se-ia pensar em menor estabilidade da versão em português. Contudo, deve-se observar que o intervalo entre as aplicações daquela pesquisa foi de apenas duas semanas, enquanto que na presente, de um mês.

Resumindo, no que concerne à precisão da versão em português dos instrumentos, pode-se dizer que ambos se mostraram confiáveis, embora a ECI tenha evidenciado melhores índices, tanto no que diz respeito à homogeneidade (consistência interna), quanto à consistência ao longo do tempo.

Quanto à validade de constructo foi verificada através de análise fatorial e validação discriminante. Como anteriormente referido, foi realizada apenas a análise fatorial com a ECI, cuja estrutura de respostas se aplica melhor a este tipo de análise.

Quanto ao QCI, o índice de correlação de Spearman de 0,47 com a ECI mostra que estes instrumentos apresentam correlação moderada que explica apenas $22 \%$ da variância, sugerindo portanto que medem constructos algo diferentes. Por outro lado, o fato da correlação não ser tão alta fala a favor da ECI que apresenta uma estrutura mais sofisticada de respostas, uma vez que estas podem ser moduladas segundo cinco alternativas e não apenas duas, como é o caso do QCI.

Conforme já referido, Malouff e Schutte (1986) desenvolveram a ECI como uma alternativa para as escalas de medida das crenças irracionais que não apresentavam qualidades psicométricas evidentes. Pretendiam que a ECI se constituísse numa contribuição mais válida do que as escalas anteriores, o que parece em parte confirmado também para a versão em português. 
A análise fatorial mostrou que a versão em português da ECI é unidimensional, encontrando-se a maioria de seus itens agrupados em torno de um fator. No entanto, não se pode utilizar em nosso meio a tradução pura e simples da original, já que o percentual, relativamente modesto de explicação da variância acumulada pelo fator $(18,89 \%$ para a amostra geral), sugere representatividade apenas aceitável dele. Ou seja, o conjunto de itens representados no fator principal estaria medindo características que também guardam associação com outros constructos, que não necessariamente as crenças irracionais.

A análise das cargas de cada item permite uma visão mais detalhada da estrutura da escala. Focalizando inicialmente as cargas fatoriais dos itens na amostra geral verifica-se que:

1) dentre os 20 itens, apenas 3 (1, 9 e 19) não apresentam a carga mínima de 0,30 , esperada para itens representativos do fator (Pasquali, 1999);

2) os Itens 9 e 19 apresentam alta saturação no segundo fator, sendo que nos dois casos a correlação é negativa. $\mathrm{O}$ Item 9 corresponde à afirmação de que "é melhor esperar do que tentar melhorar uma situação ruim da vida"; enquanto segundo o 19, "é melhor ignorar os problemas pessoais do que tentar resolvê-los". Isto é, nos dois casos as afirmações relacionam-se a uma atitude passiva frente ao infortúnio, com forte matiz depressivo. Conforme já referido, têm sido verificadas associações das crenças irracionais com depressão, embora na versão original estes itens tenham apresentado maior correlação no fator principal;

3) o Item 1 apresenta carga fatorial próxima do limite mínimo no primeiro fator e também correlaciona-se negativamente com o segundo, mas também aqui de forma insuficiente Quanto ao seu conteúdo (para ser uma pessoa de valor preciso ser totalmente competente em tudo o que faço), necessita provavelmente de uma adaptação na sua formulação, deixando mais explícita a crença irracional subjacente;

4) as cargas dos demais itens oscilam entre 0,36 (Item 2) e 0,62 (Item 8), o que sugere boa representação comportamental deles (Pasquali, 1999);

5) deve-se observar, no entanto, que no Item 4 , a maior carga fatorial encontra-se registrada no Fator 2, sendo que neste caso a correlação é negativa. Como no caso dos Itens 9 e 19, o conteúdo parece relacionado a um estado depressivo em que há uma tendência a generalizar os aspectos negativos das situações (a maioria das pessoas que têm sido injustas comigo são em geral ruins).

Nas subamostras por sexo, verifica-se a mesma estrutura unidimensional, sendo que na amostra masculina:

1) 18 dos 20 itens apresentam carga fatorial igual ou superior a 0,34 ;
2) os Itens 9 e 19 não apresentam carga suficiente no primeiro fator mas apresentam alta correlação negativa no segundo fator, como verificado para a amostra geral;

3) os Itens 15 e 18 também apresentam altas cargas positivas no Fator 2, o que pode ser motivo de dificuldade quanto à compreensão de sua representatividade para sujeitos do sexo masculino.

4) os Itens 5 e 11 têm alta representação negativa no Fator 2. Como no caso dos Itens 9 e 19 estariam relacionados a sentimento de impotência frente à inexorabilidade dos fatos, o que é característico de estados depressivos (5 algumas de minhas maneiras de agir são tão arraigadas que nunca poderia mudá-las; 11 - muitos eventos de meu passado me influenciam tão intensamente que é impossível mudar).

Quanto à amostra feminina, encontra-se mais alinhada com a amostra geral no que respeita às cargas fatoriais dos itens, pois:

1) além dos Itens 1,9 e 19, apenas o Item 2 não atingiu carga fatorial mínima de 0,30 no primeiro fator, apesar do valor estar próximo $(0,29)$;

2) o Item 4 apresenta maior covariância com o Fator 2 (17\% contra 15\% no Fator 1), em acordo com a amostra geral;

3) o Item 10 apresenta covariâncias muito semelhantes com os dois fatores, respectivamente $10,4 \%$ e $10,2 \%$, embora no primeiro fator a associação seja positiva e no segundo negativa.

Com base na análise realizada, sugere-se que sejam retidos para a versão brasileira da escala os itens que apresentaram carga fatorial mínima de 0,30 no primeiro fator de ao menos uma das amostras por sexo e na amostra geral. De acordo com este critério comporiam a escala os seguintes Itens: 2 , $3,5,6,7,8,10,11,12,13,14,15,16,17,18$ e 20.

Tomados em conjunto, estes itens detêm representação comportamental aceitável do constructo de crenças irracionais, constituindo-se em medida válida delas, ao menos para populações de estudantes universitários que apresentem perfil semelhante ao da amostra estudada.

Quanto aos demais itens, necessitarão de revisão mais pormenorizada de seus conteúdos, podendo sofrer adaptações no seu enunciado, como é o caso do Item 1 que apresenta saturação adequada para a amostra masculina e valor próximo de 0,30 para as amostras feminina $(0,28)$ e geral $(0,29)$; ou ainda serem totalmente modificados, como os Itens 9 e 19, com altas cargas no segundo fator dos três grupos amostrais e o Item 4, que apresentou alta covariação com o Fator 2 na amostra feminina e geral. Uma análise empírica dos itens (dificuldade e discriminação) poderá complementar o estudo ora apresentado e contribuir mais especificamente para a identificação dos itens a serem adaptados. 
Quanto à validade discriminante, verifica-se índice de correlação superior entre o QSG e a ECI se comparado ao observado entre o QSG e o QCI. Mas, ainda assim, a correlação é fraca $(0,36)$. Com base neste índice, é possível cogitar que as crenças irracionais contribuam apenas em situações específicas de sintomas atuais de stress psíquico, desejo de morte, desconfiança no próprio desempenho, distúrbio do sono e distúrbio psicossomático, componentes do QSG. E neste sentido, os resultados estariam sugerindo que as crenças irracionais e os aspectos de saúde geral, medidos pelo QSG, constituem expressões de fenômenos psíquicos independentes que detêm entre si índices modestos de associação.

\section{Conclusões}

Pode-se dizer deste primeiro estudo de validação da versão em português da ECI e do QCI que, embora os dois instrumentos apresentem boas qualidades psicométricas, a ECI demonstrou melhores índices de precisão (tanto de consistência interna, quanto de teste e reteste), além de sua estrutura (respostas em escala Likert) facilitar a análise fatorial, que oferece uma medida mais segura da validade de constructo.

Para sujeitos com o perfil semelhante aos da amostra estudada (jovens, universitários, de ambos os sexos), a versão brasileira deve integrar os 16 itens que apresentaram maior representatividade do constructo, sendo que os demais deverão sofrer adaptações e modificações.

O conjunto dos itens modificados deverá ser novamente submetido à investigação empírica, para o estabelecimento de sua validade.

Para populações com características diferentes como, por exemplo, pacientes de serviços voltados para a saúde mental, distúrbios psicossomáticos, ou idosos, novas pesquisas deverão ser realizadas para a definição da forma mais viável da escala. Recomenda-se, portanto, a continuidade das investigações em torno da validação da ECI como instrumento de avaliação promissor de crenças irracionais para sujeitos brasileiros. Quanto à validade de critério, novas pesquisas, envolvendo outras medidas, devem ser realizadas.

\section{Referências}

Anastasi, A. \& Urbina, S. (2000). Testagem psicológica (M. A. V. Veronese, Trad.). Porto Alegre: Artes Médicas. (Original publicado em 1997)

Calais, S. L. (1997). Crenças irracionais e habilidade social em universitários. Dissertação de Mestrado não-publicada, Curso de Pós-Graduação em Psicologia, Pontifícia Universidade Católica de Campinas. Campinas, SP.

Ellis, A. (1993). Reflections on rational-emotive therapy. Em M. J. Mahoney (Org.), Cognitive and constructive psychotherapies: Theory research and practice (pp. 69-73). New York: Springer Publishing Company.

Ellis, A. (1995). Changing rational-emotive therapy (RET) to rational emotive behavior therapy (REBT). Journal of Rational-Emotive and CognitiveBehavior Therapy, 13, 85-89.

Malouff, J. M. \& Schutte, N. S. (1986). Development and validation of a measure of irrational belief. Journal of Consulting and Clinical Psychology, 54(6), 860-862.

Malouff, J. M., Valdenegro, J. \& Schutte, N. S. (1987). Further validation of a measure of irrational belief. Journal of Rational-Emotive and CognitiveBehavior Therapy, 5, 189-193.

Mayhew, R. \& Edelman, R. J. (1989). Self-esteem, irrational beliefs and coping strategies in relation to eating problems in a non-clinical population. Personality and Individual Differences, 10, 581-584.

Newmark, C. S., Frerking, R. A., Cook, L. \& Newmark, L. (1973). Endorsement of Ellis' Irrational Beliefs as a function of psychopathology. Journal of Clinical Psychology, 29, 300-302.

Pasquali, L. (Org.) (1999). Instrumentos psicológicos: Manual prático de elaboração. Brasília: LabPAM/IBAPP.

Pasquali, L., Gouveia, V. V., Andriola, W. B., Miranda, F. J. \& Ramos, A. L. M. (1996). Questionário de saúde geral de Goldberg: Manual técnico QSG (adaptação brasileira). São Paulo: Casa do Psicólogo.

Pinto, W. N. R. (1996). Stress e qualidade de vida de pacientes com vitiligo. Dissertação de Mestrado não-publicada, Curso de Pós-Graduação em Psicologia, Pontifícia Universidade Católica da Campinas. Campinas, SP.

Rorer, L. G. (1989a). Rational-emotive theory: An integrated psychological and philosophical basis. Cognitive Therapy and Research, 13, 475-492.

Rorer, L. G. (1989b). Rational-emotive theory: Explication and evaluation. Cognitive Therapy and Research, 13, 531-548.

Schutte, N. S. \& Malouff, J. M. (1995). Irrational Belief Scale. Em N. S Schutte \& J. M. Malouff (Orgs.), Sourcebook of adult assessment strategies (pp. 432-435). New York: Plenum Press.

Shorkey, C. T. \& Whiteman, V. L. (1977). Development of the Rational Behavior Inventory: Initial validity and reliability. Educational and Psychological Measurement, 37, 527-534.

Templeman, T. L. (1990). Relationship of M. S. Belief Scale scores to depression and anxiety in hospitalized patients. Journal of RationalEmotive and Cognitive-Behavior Therapy, 8, 267-274.

Warren, R. \& Zgourides, G. (1989). Further validity and normative data for the Malouff and Schutte Belief Scale. Journal of Rational-Emotive and Cognitive-Behavior Therapy, 7, 167-172.

Wertheim, E. \& Poulakis, Z. (1992). The relationships among the General Attitude and Belief Scale, other dysfunctional cognition measures, and depressive or bulimic tendencies. Journal of Rational-Emotive and Cognitive-Behavior Therapy, 10, 219-233.

\section{Sobre os autores}

Elisa Medici Pizão Yoshida é Doutora em Ciências (Psicologia) pela Universidade de São Paulo, Pós-Doutorado na Universidade de Montréal, Docente do Programa de Pós-Graduação em Psicologia da Pontifícia Universidade Católica de Campinas e psicoterapeuta.

Fernando Antonio Basile Colugnati é Mestre em Estatística pela Universidade Estadual de Campinas com ênfase em Métodos Multivariados, estatístico pesquisador do Programa de PósGraduação em Nutrição e Saúde da Escola Paulista de Medicina/UNIFESP. 